\title{
Ultrasound assessment of gastric contents in emergency patients examined in the full supine position: an appropriate composite ultrasound grading scale can finally be proposed
}

\author{
Lionel Bouvet $^{1} \cdot$ Dominique Chassard $^{1}$
}

Received: 11 December 2019 / Accepted: 17 December 2019 / Published online: 18 December 2019

C) Springer Nature B.V. 2019

Point-of-care ultrasonography allows bedside ultrasoundguided management of clinical issues such as acute respiratory and hemodynamic failures [1], undifferentiated shocks or shocks related to trauma using FAST ultrasound [2, 3]. Thus, it has become a major diagnosis and assessment tool available to the anesthesiologist facing critical situations in the operating room or in critical care unit.

Pulmonary aspiration of gastric contents remains one of the leading causes of mortality and morbidity related to anesthesia; consequently, every patient should be assessed for the risk of aspiration prior to anesthesia [4]. Some risk factors for regurgitation and aspiration are well known by the anesthesiologists, especially those related to lower esophageal sphincter failure or to the increase in gastric content volume due to gastrointestinal obstruction. Nevertheless, in many circumstances, clinical assessment of the risk of aspiration remains difficult and uncertain. In particular, the minimal fasting duration ensuring low risk of aspiration in emergency patients remains unclear, leading the ASA guidelines to state that current fasting guidelines do not apply to emergency patients [5]. In fact, gastric content status depends on several factors, including the type and amount of food, the caloric value of the last meal, preexisting gastroparesis, the administration of some analgesic drugs delaying gastric emptying during the preoperative period, the level of preoperative pain and stress affecting gastric emptying. Therefore, point-of-care gastric ultrasound has become a useful tool for the preoperative assessment of gastric content and volume, helping the anesthesiologist to assess the

Lionel Bouvet

lionel.bouvet@chu-lyon.fr

1 Department of Anesthesiology and Intensive Care, Femme-Mère-Enfant Hospital, Groupement Hospitalier Est, Hospices Civils de Lyon, 59 boulevard Pinel, Bron, 69500 Lyon, France risk of regurgitation and aspiration, in addition to the clinical assessment of the risk of aspiration.

Gastric ultrasound, actually antral ultrasound, was first described for the determination of gastric emptying of a test meal in patients suffering from gastrointestinal diseases. This technique implies repeated measurements of the antral cross-sectional area to calculate gastric emptying rate that highly correlates to the rate provided by gastric scintigraphy [6]. On the other hand, preoperative ultrasound diagnosis of gastric content status can only be based on the appropriate and careful interpretation of a single real-time ultrasound examination of the antrum. The reliability of gastric ultrasound for the preoperative assessment of gastric contents and volume has been the subject of several studies published these 10 last years.

Three approaches for the interpretation of gastric ultrasound have been described that are actually complementary. A first approach is to focus on the qualitative appearance of gastric antrum content, the visualization of solids or thick fluids into the antrum corresponding to high-risk gastric contents [7]. As regards clear fluids, the visualization of anechoic fluid content in the antrum in the full supine position corresponds to increased gastric fluid volume, $>250 \mathrm{ml}$ in $50 \%$ of the cases, while empty antrum in both the full supine and the right lateral decubitus positions corresponds to an empty, 'low-risk', stomach [8, 9]. This qualitative assessment may be completed by the measurement of the antral cross-sectional area in the right lateral decubitus position for the calculation of the volume of clear fluid content, to determine whether it is $\geq$ or $<1.5 \mathrm{ml} / \mathrm{kg}$ [8]. Another approach was to determine the cut-off value of the antral cross-sectional area measured in the supine position with the head of the bed elevated to $45^{\circ}$ to distinguish between 'low-risk' and 'high-risk' stomach. This cut-off value was $340 \mathrm{~mm}^{2}$ in a study conducted in 183 patients, with a sensitivity of $91 \%$, a specificity of $71 \%$ and a negative predictive value of $94 \%$, which means that the likelihood of low risk stomach 
is $94 \%$ if antral area is $<340 \mathrm{~mm}^{2}$ in the semi-upright position [10]. These approaches raise several issues regarding their applicability in the setting of emergency surgery. The mathematical model applies only to clear fluids contents, and this mathematical calculation of gastric fluid volume as well as the qualitative analysis of fluid contents requires turning the patient in the right lateral decubitus position, what was not feasible in a quarter of emergency patients in a previous study [11]. The determination of the cut-off value of the antral cross-sectional area was performed in elective and emergency patients lying in the semi-upright position and not in the full supine position, furthermore using gastric suctioning as a comparator to measure the actual gastric content volume, a method that has its own limitations. Hence, for emergency patients lying in the full supine position who cannot be placed in the semi-upright position for ultrasound examination (e.g. some trauma patients), qualitative assessment may remain incomplete and the diagnosis value of the antral area for quantitative or semi-quantitative assessment is undetermined.

In this issue of JCMC, Okada et al. [12] determined the cut-off value of the antral cross sectional area measured in emergency patients lying in the full supine position for the diagnosis of high-risk stomach, using computed tomography as a comparator to measure the actual gastric content volume and to assess the type of content. This non-invasive imaging technique has been used as comparator of gastric ultrasound in critical care patients and may reasonably be considered as providing accurate calculation of gastric content volume [13], though in the study by Okada et al., the median interval time between computed tomography and ultrasound examination was somewhat long [12]. The cut-off value of the antral cross-sectional area for the diagnosis of gastric fluid volume $>1.5 \mathrm{ml} / \mathrm{kg}$ and/or solid/thick fluid contents was $3.01 \mathrm{~cm}^{2}$. This cut-off value applies for the detection of a full stomach according to a definition that is widely currently admitted, based to the fact that gastric fluid volume $>1.5 \mathrm{ml} / \mathrm{kg}$ and/or solid/ thick fluid gastric contents have rarely been reported in elective fasted patients [14]. This cut-off value is particularly appropriate for use in clinical practice, in emergency patients in whom the stomach is rarely filled with only clear fluids. This cut-off value is slightly less than those that have been calculated in elective and emergency patients $\left(340 \mathrm{~mm}^{2}\right.$ or $\left.3.4 \mathrm{~cm}^{2}\right)$ and in critical care patients $\left(3.6 \mathrm{~cm}^{2}\right)$ lying in the semi-upright position, with furthermore decreased diagnosis performance [10, 13]. This is not fully surprising because patient body position significantly affects the reliability of qualitative and quantitative ultrasound assessments [15]. For example, the sensitivity of qualitative diagnosis of gastric fluid volume $>1.5 \mathrm{ml} /$ $\mathrm{kg}$ increases from 12 to $71 \%$ when elevating the head of the bed from $0^{\circ}$ to $45^{\circ}$ [15]. With a sensitivity of $85 \%$ and a specificity of $53 \%$, the cut-off value calculated by Okada et al. is not sufficient to ensure reliable diagnosis of full or empty stomach in emergency patients lying in the full supine position. So, how can this value be used in clinical practice?

As mentioned above, qualitative and quantitative approaches provide complementary information and should actually be used together for optimal interpretation of ultrasound examination of gastric antrum. The concept of composite ultrasound grading scale combining the cut-off value of the antral area to the qualitative examination of the gastric antrum has recently emerged [11]. Such a composite scale has been described in elective and emergency patients lying in the supine position with the head of the bed elevated to $45^{\circ}$ and in term pregnant women, using appropriate cutoff values of antral area $[11,16]$. The main advantages of composite scales are, first, fast ultrasound assessment of gastric contents status, and second, increased diagnosis performance of antral ultrasound compared to qualitative and quantitative approaches used separately $[15,16]$. In emergency patients lying in the full supine position, high risk stomach may be defined by the visualization of any contents (fluid or solid) in the gastric antrum, while low-risk stomach may be defined by empty antrum with antral area $<3.01 \mathrm{~cm}^{2}$. Empty antrum and antral area $>3.01 \mathrm{~cm}^{2}$ may correspond to intermediate risk stomach, and should be interpreted according to the clinical assessment of risk factors of regurgitation and aspiration. A clinical algorithm for ultrasound assessment of gastric content volume in patients examined in the full supine position can be proposed (Fig. 1), whose reliability remains to be assessed, even though it has previously been established that a two-steps clinical algorithm (qualitative assessment followed by volume calculation of clear fluid contents) is highly specific and sensitive for the diagnosis of a full or empty stomach [17].

In conclusion, the work by Okada et al. [12] contributes to clarify the interpretation of the ultrasound examination of the gastric antrum for the diagnosis of low-risk vs highrisk stomach in emergency patients in the full supine position with mixed gastric contents. Their results should be applied as part of composite ultrasound grading scale that requires nevertheless further assessments to rate its diagnosis performance and to improve the diagnosis interpretation of intermediate gastric content. We still have some work to do to improve the reliability and accuracy of point-of-care gastric ultrasound. 
Fig. 1 Clinical algorithm for ultrasound diagnosis of gastric contents in patients lying in the full supine position. *The algorithm in patients examined in the semi-upright $\left(45^{\circ}\right)$ position is the same as this described in patients lying in the full supine position, but using cut-off value of antral area of $340 \mathrm{~mm}^{2}$ and with increased reliability [15]

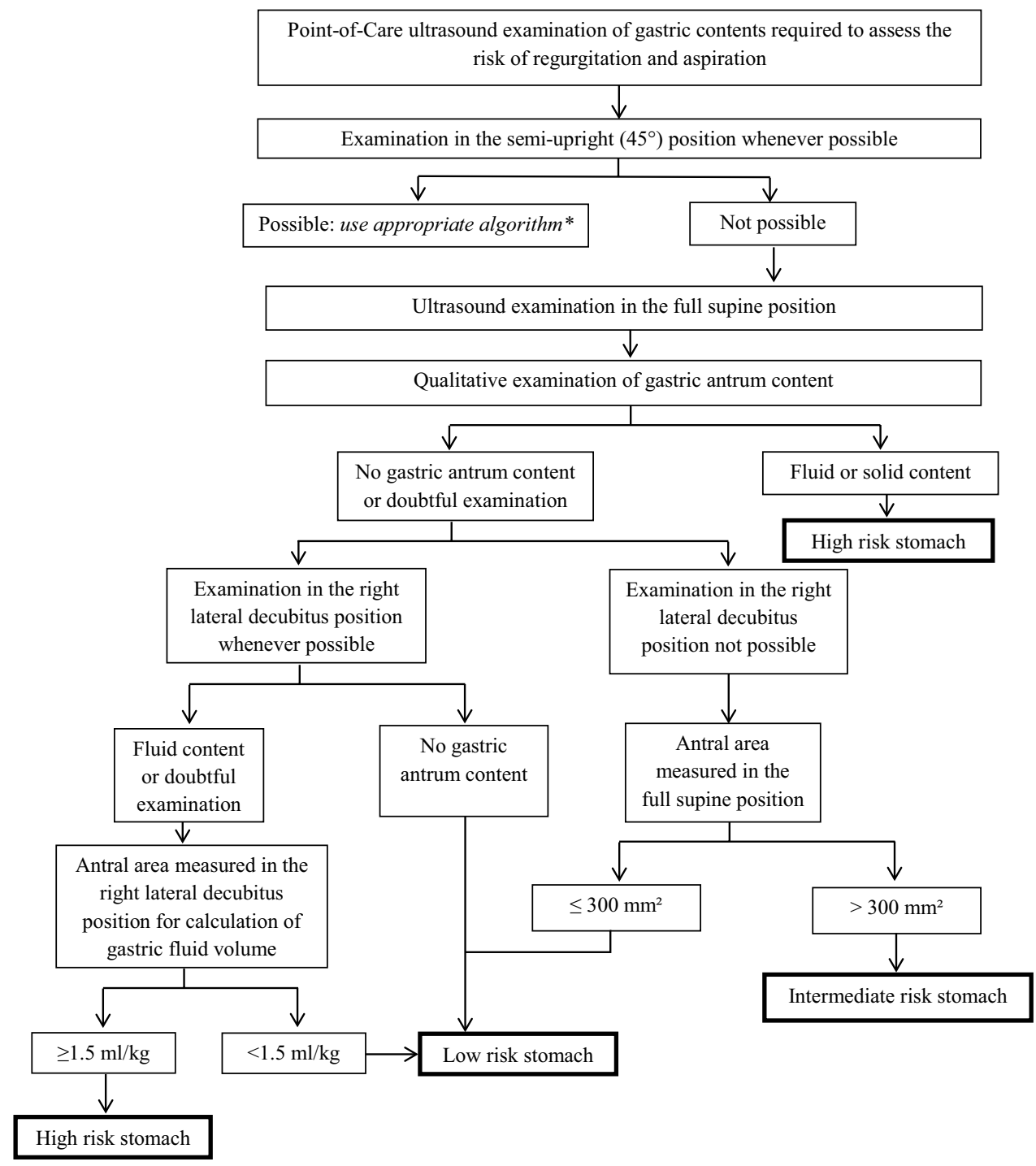

\section{Compliance with ethical standards}

Conflict of interest All authors declare that they have no conflict of interest.

\section{References}

1. Moore CL, Copel JA. Point-of-care ultrasonography. N Engl J Med. 2011;364:749-57.

2. Jones AE, Tayal VS, Sullivan DM, Kline JA. Randomized, controlled trial of immediate versus delayed goal-directed ultrasound to identify the cause of nontraumatic hypotension in emergency department patients. Crit Care Med. 2004;32:1703-8.

3. Melanson SW, Heller M. The emerging role of bedside ultrasonography in trauma care. Emerg Med Clin N Am. 1998;16:165-89.
4. Cook TM, Woodall N, Frerk C. Major complications of airway management in the UK: results of the Fourth national audit project of the royal college of anaesthetists and the difficult airway society. Part 1: anaesthesia. Br J Anaesth. 2011;106:617-31.

5. Practice guidelines for preoperative fasting and the use of pharmacologic agents to reduce the risk of pulmonary aspiration: application to healthy patients undergoing elective procedures: an updated report by the American society of anesthesiologists task force on preoperative fasting and the use of pharmacologic agents to reduce the risk of pulmonary aspiration. Anesthesiology. 2017;126:376-93.

6. Bolondi L, Santi V, Bortolotti M, Li Bassi S, Turba E. Correlation between scintigraphic and ultrasonographic assessment of gastric emptying. Gastroenterology. 1986;90:1349.

7. Perlas A, Van de Putte P, Van Houwe P, Chan VW. I-AIM framework for point-of-care gastric ultrasound. Br J Anaesth. 2015;116:7-11.

8. Perlas A, Mitsakakis N, Liu L, Cino M, Haldipur N, Davis L, et al. Validation of a mathematical model for ultrasound assessment of gastric volume by gastroscopic examination. Anesth Analg. 2013;116:357-63. 
9. Perlas A, Davis L, Khan M, Mitsakakis N, Chan VW. Gastric sonography in the fasted surgical patient: a prospective descriptive study. Anesth Analg. 2011;113:93-7.

10. Bouvet L, Mazoit JX, Chassard D, Allaouchiche B, Boselli E, Benhamou D. Clinical assessment of the ultrasonographic measurement of antral area for estimating preoperative gastric content and volume. Anesthesiology. 2011;114:1086-92.

11. Bouvet L, Desgranges FP, Aubergy C, Boselli E, Dupont G, Allaouchiche B, et al. Prevalence and factors predictive of full stomach in elective and emergency surgical patients: a prospective cohort study. Br J Anaesth. 2017;118:372-9.

12. Okada Y, Toyama H, Kamata K, Yamauchi M. A clinical study comparing ultrasound-measured pyloric antrum cross-sectional area to computed tomography-measured gastric content volume to detect high-risk stomach in supine patients undergoing emergency abdominal surgery. J Clin Monit Comput. 2019. https://doi. org/10.1007/s10877-019-00438-1.

13. Hamada SR, Garcon P, Ronot M, Kerever S, Paugam-Burtz C, Mantz J. Ultrasound assessment of gastric volume in critically ill patients. Intensiv Care Med. 2014;40:965-72.
14. Van de Putte P, Perlas A. The link between gastric volume and aspiration risk. In search of the Holy Grail? Anaesthesia. 2018;73:274-9.

15. Bouvet L, Barnoud S, Desgranges FP, Chassard D. Effect of body position on qualitative and quantitative ultrasound assessment of gastric fluid contents. Anaesthesia. 2019;74:862-7.

16. Roukhomovsky M, Zieleskiewicz L, Diaz A, Guibaud L, Chaumoitre K, Desgranges FP, et al. Ultrasound examination of the antrum to predict gastric content volume in the third trimester of pregnancy as assessed by MRI: a prospective cohort study. Eur J Anaesthesiol. 2018;35:379-89.

17. Kruisselbrink R, Gharapetian A, Chaparro LE, Ami N, Richler $\mathrm{D}$, Chan VWS, et al. Diagnostic accuracy of point-of-care gastric ultrasound. Anesth Analg. 2019;128:89-95.

Publisher's Note Springer Nature remains neutral with regard to jurisdictional claims in published maps and institutional affiliations. 